\title{
HYDJET++ model for the ultra-relativistic heavy-ion collisions: new results and developments
}

\author{
S.V. Petrushanko*, G.Kh. Eyyubova, V.L. Korotkikh, I.P. Lokhtin, L.V. Malinina, \\ E.N. Nazarova, A.A. Sidorova, A.M. Snigirev, E.E. Zabrodin \\ Skobeltsyn Institute of Nuclear Physics, Lomonosov Moscow State University, \\ Moscow, Russian Federation \\ E-mail: Serguei.Petrouchankodcern.ch
}

\section{L.V. Bravina}

The Department of Physics, University of Oslo

Oslo, Norway

\begin{abstract}
HYDJET++ model combines the description of soft processes with the treatment of hard partons propagating medium. The model is employed for the analysis of $\mathrm{PbPb}$ collisions at $\mathrm{LHC}$ energies, particularly, the azimuthal anisotropy phenomena, flow, femtoscopy, hard probes. The influence of geometric and dynamical anisotropies on the development of flow harmonics and, simultaneously, on the second- and third-order oscillations of femtoscopy radii were studied. The interplay of soft hydro-like processes and jets is able to describe the violation of the mass hierarchy of meson and baryon elliptic and triangular flows at transverse momentum $p_{\mathrm{T}}>2 \mathrm{GeV} / c$, the falloff of the anisotropic flow harmonics at intermediate transverse momenta, and the worsening of the number-of-constituent-quark (NCQ) scaling of elliptic/triangular flow at LHC compared to RHIC energies. The cross-talk of elliptic and triangular flows leads to emergence of higher order harmonics in the model and to appearance of ridge structure in dihadron angular correlations in a broad pseudorapidity range. Recently, the model was further extended to describe quantitatively the event-by-event fluctuations of the anisotropic flow. The model calculations agree well with the experimental data.
\end{abstract}

The 39th International Conference on High Energy Physics (ICHEP2018)

4-11 July, 2018

Seoul, Korea

\footnotetext{
* Speaker.

${ }^{\dagger}$ Also at Joint Institute for Nuclear Researches, Dubna, Russian Federation

¥Also at The Department of Physics, University of Oslo, Oslo, Norway and National Research Nuclear University "MEPhI" (Moscow Engineering Physics Institute), Moscow, Russian Federation
} 


\section{Introduction. HYDJET++ model}

The basic features of the HYDJET++ model are described in [1]. The model combines two components corresponding to soft and hard processes. The soft part of the model has no evolution stage from the initial state until hadronization, but rather represents a thermal hadron production already at the freeze-out hypersurface in accordance with the prescriptions of ideal hydrodynamics adapted from the event generator FAST MC [2]. The hard part of the model is based on PYTHIA [3] and PYQUEN [丹] generators, which simulate parton-parton collisions, parton radiative energy loss, and hadronization. The latest available via internet version of HYDJET++ is 2.3 [阿].

\section{Results}

It was demonstrated that the HYDJET++ model can describe the properties of the hadronic state created in AuAu collisions at RHIC at center-of-mass energy per nucleon pair $\sqrt{s_{\mathrm{NN}}}=200$ $\mathrm{GeV}$ [1]. We have applied HYDJET++ with tuned input parameters to reproduce the LHC data from $\mathrm{PbPb}$ collisions at $\sqrt{s_{\mathrm{NN}}}=2.76 \mathrm{TeV}$. HYDJET++ allows us to reproduce the LHC data on centrality and pseudorapidity dependence of charged particle multiplicity, tranverse momentum $p_{\mathrm{T}}$-spectra, nuclear modification factor $R_{\mathrm{AA}}$ (up to $p_{\mathrm{T}} \sim 100 \mathrm{GeV} / c$ ), $\pi^{ \pm} \pi^{ \pm}$correlation radii in central $\mathrm{PbPb}$ collisions, and $p_{\mathrm{T}^{-}}$and $\eta$-dependencies of the elliptic flow coefficient $v_{2}$ [6].

The effects of possible non-elliptic shape of the initial overlap of the colliding nuclei are implemented in HYDJET++ by the modulation of the final freeze-out hypersurface with the appropriate fitting triangular coefficient 伊. This modulation is not correlated with the direction of the impact parameter, and two independent "strong" lower azimuthal harmonics, elliptical $v_{2}$ and triangular $v_{3}$, being obtained as a result. Interference between $v_{2}$ and $v_{3}$ generates as "overtones" both even and odd higher azimuthal harmonics, $v_{4}, v_{5}, v_{6}$, etc. This mechanism allows HYDJET++ to reproduce the LHC data on $p_{\mathrm{T}^{-}}$and centrality dependencies of the anisotropic flow coefficients $v_{n}$ (n=2 $\div 4$ ) up to $p_{\mathrm{T}} \sim 5 \mathrm{GeV} / c$ and $40 \%$ centrality, and also the basic trends for pentagonal $v_{5}$ and hexagonal $v_{6}$ flows. Our study [8] favors the idea that basic features of the hexagonal flow $v_{6}$ can be understood in terms of the interplay of elliptic and triangular flows.

The analysis of dihadron angular correlations measured in $\mathrm{PbPb}$ collisions at $\sqrt{s_{\mathrm{NN}}}=2.76$ $\mathrm{TeV}$ with the HYDJET++ model was done [9]. It was shown that the structure called a "ridge" in dihadron angular correlations could appear just as the interplay of $v_{2}$ and $v_{3}$.

The triangular flow $v_{3}$ of charged inclusive and identified hadrons is studied within the HYDJET++ model in $\mathrm{PbPb}$ collisions at $\sqrt{s_{\mathrm{NN}}}=2.76 \mathrm{TeV}$ and centralities $0-50 \%$ [10]. Mass ordering effect is obtained: the flow of mesons is stronger than that of baryons. The interplay of hard and soft processes leads to breaking of the mass ordering of triangular flows, because jet particles start to dominate spectra of heavy hadrons at larger $p_{T}$ compared to those of light hadrons. The resonance decays modify the spectra towards the number-of-constituent-quark (NCQ) scaling fulfillment for $v_{3}$, whereas jets are the main source of the scaling violation at energies of LHC.

Event-by-event harmonic flow coefficients measured in $\mathrm{PbPb}$ collisions at $\sqrt{s_{\mathrm{NN}}}=2.76 \mathrm{TeV}$ are interpreted within the HYDJET++ model in [11]. It is shown that the simple modification of the model via introducing the distribution over spatial anisotropy parameters permits HYDJET++ 
to reproduce both elliptic and triangular flow fluctuations and related to it eccentricity fluctuations of the initial state at the LHC energy.

The phenomenological analysis of charmed meson and charmonium production in $\mathrm{PbPb}$ collisions at $\sqrt{s_{\mathrm{NN}}}=2.76 \mathrm{TeV}$ was done within the HYDJET++ model [12]. The significant part of $D$ mesons, unlike $J / \psi$ mesons, seems to be in a kinetic equilibrium with hot hadronic matter.

PYQUEN model [4] was applied to simulate medium-modified inclusive jet production at $\sqrt{s_{\mathrm{NN}}}=2.76 \mathrm{TeV}[13]$. The contribution from wide-angle radiative energy loss dominates.

We have studied the influence of geometric and dynamical anisotropies on the development of flow harmonics and, simultaneously, on the second- and third-order oscillations of femtoscopy radii [14]. It was shown that the merely geometric anisotropy provides the results which anticorrelate with the experimental observations of either $v_{2}$ (or $v_{3}$ ) or second-order (or third-order) oscillations of the femtoscopy radii. Decays of resonances significantly increase the emitting areas but do not change the phases of the radii oscillations. In contrast to the spatial deformations, the dynamical anisotropy alone provides the correct qualitative description of the flow and the femtoscopy observables simultaneously. However, one needs both types of the anisotropy to match quantitatively the experimental data.

The various mechanisms of $B^{ \pm}$meson suppression have been analyzed recently with HYDJET++ model [15]. It has been shown that the contributions of nuclear shadowing and jet quenching into $B^{ \pm}$meson suppression are comparable at $p_{\mathrm{T}} \sim 10 \mathrm{GeV} / c$. Then the relative contribution of jet quenching gets stronger with increasing $p_{\mathrm{T}}$, and totally dominates at $p_{\mathrm{T}}>30 \mathrm{GeV} / c$. Since $B^{ \pm}$ meson suppression factor $R_{\mathrm{AA}}$ due to jet quenching (nuclear shadowing) decreases (increases) with $p_{\mathrm{T}}$, the interplay between the two effects results in a weak (roughly constant) $p_{\mathrm{T}}$-dependence of $R_{\mathrm{AA}}$. This observation is consistent with the trend seen in the CMS data [16].

\section{Conclusions}

The combination of the soft and hard components in HYDJET++ model allows us to describe a number of physical observables at ultra-relativistic heavy-ion collisions. Further modificaions of the model can open new horizons for understanding of the hot matter and the quark-gluon plasma.

\section{Acknowledgments}

The speaker would like to thank the Organizers of ICHEP 2018 for the very generous welcome in Korea. We thank our colleagues from the CMS, ALICE and ATLAS collaborations for fruitful cooperation.

This work was supported by Russian Foundation for Basic Research (grant 18-02-00155).

\section{References}

[1] I.P. Lokhtin, L.V. Malinina, S.V. Petrushanko, A.M. Snigirev, I. Arsene, and K. Tywoniuk, Heavy ion event generator HYDJET++ (HYDrodynamics plus JETs), Comput. Phys. Commun. 180 (2009) 779 [hep-ph/0809.2708] 
[2] N.S. Amelin, R. Lednicky, I.P. Lokhtin, L.V. Malinina, A.M. Snigirev, Iu.A. Karpenko, Yu.M. Sinyukov, I. Arsene, and L. Bravina, Fast hadron freeze-out generator. II. Noncentral collisions, Phys. Rev. C 77 (2008) 014903 [hep-ph/0711.0835]

[3] T. Sjostrand, S. Mrenna, and P. Skands, PYTHIA 6.4 Physics and Manual, J. High Energy Phys. 05 (2006) 026 [hep-ph/ 0603175$]$

[4] I.P. Lokhtin and A.M. Snigirev, A model of jet quenching in ultrarelativistic heavy ion collisions and high-p $\mathrm{T}_{\mathrm{T}}$ hadron spectra at RHIC, Eur. Phys. J. C 45 (2006) 211 [hep-ph / 0506189 ]

[5] http://lokhtin.web.cern.ch/lokhtin/hydjet++

[6] I.P. Lokhtin, A.V. Belyaev, L.V. Malinina, S.V. Petrushanko, E.P. Rogochaya, A.M. Snigirev, Hadron spectra, flow and correlations in PbPb collisions at the LHC: interplay between soft and hard physics, Eur. Phys. J. C 72 (2012) 2045 [hep-ph/ 01204 . 4820]

[7] L.V. Bravina, B.H. Brusheim Johansson, G.Kh. Eyyubova, V.L. Korotkikh, I.P. Lokhtin, L.V. Malinina, S.V. Petrushanko, A.M. Snigirev, E.E. Zabrodin, Higher harmonics of azimuthal anisotropy in relativistic heavy-ion collisions in HYDJET++ model, Eur. Phys. J. C 74 (2014) 2807 [hep-ph/01311.7054]

[8] L.V. Bravina, B.H. Brusheim Johansson, G.Kh. Eyyubova, V.L. Korotkikh, I.P. Lokhtin, L.V. Malinina, S.V. Petrushanko, A.M. Snigirev, E.E. Zabrodin, Hexagonal flow $v_{6}$ as a superposition of elliptic $v_{2}$ and triangular $v_{3}$ flows, Phys. Rev. C 89 (2014) 024909 [hep-ph/1311 . 074 7]

[9] G. Eyyubova, V.L. Korotkikh, I.P. Lokhtin, S.V. Petrushanko, A.M. Snigirev, L.V. Bravina, E.E. Zabrodin, Angular dihadron correlations as an interplay between elliptic and triangular flows, Phys. Rev. C 91 (2015) 064907 [hep-ph / 1411 . 4487]

[10] J. Crkovská, J. Bielčík, L. Bravina, B.H. Brusheim Johansson, E. Zabrodin, G. Eyyubova, V.L. Korotkikh, I.P. Lokhtin, L.V. Malinina, S.V. Petrushanko, A.M. Snigirev, Influence of jets and decays of resonances on the triangular flow in ultrarelativistic heavy-ion collisions, Phys. Rev. C 95 (2017) 014910 [hep-ph/1603.09621]

[11] L.V. Bravina, E.S. Fotina, V.L. Korotkikh, I.P. Lokhtin, L.V. Malinina, E.N. Nazarova, S.V. Petrushanko, A.M. Snigirev, E.E. Zabrodin, Anisotropic flow fluctuations in hydro-inspired freeze-out model for relativistic heavy ion collisions, Eur. Phys. J. C 75 (2015) 588 [hep-ph/1509.02692]

[12] I.P. Lokhtin, A.V. Belyaev, G.Kh. Eyyubova, G. Ponimatkin, E.Yu. Pronina, Charmed meson and charmonium production in PbPb collisions at the LHC, J. Phys. G 43 (2016) 125104 [hep-ph/11601.00799]

[13] I.P. Lokhtin, A.A. Alkin, A.M. Snigirev, On jet structure in heavy ion collisions Eur. Phys. J. C 75 (2015) 452 [hep-ph/1410.0147]

[14] L.V. Bravina, I.P. Lokhtin, L.V. Malinina, S.V. Petrushanko, A.M. Snigirev, E.E. Zabrodin, Dynamical vs geometric anisotropy in relativistic heavy-ion collisions: which one prevails? Eur. Phys. J. A 53 (2017) 219 [hep-ph/1709.08602]

[15] I.P. Lokhtin and A.A. Sidorova, Mechanisms of B meson suppression in ultrarelativistic heavy ion collisions, J. Exp. Theor. Phys. (in press)

[16] CMS Collaboration, Measurement of the $B^{ \pm}$meson nuclear modification factor in PbPb Collisions at $\sqrt{s_{\mathrm{NN}}}=5.02$ TeV. Phys. Rev. Lett. 119 (2017) 152301 [hep-ph/1705. 04727] 